\title{
DANUBIO - a new drug-eluting balloon for the treatment of side branches in bifurcation lesions: six-month angiographic follow-up results of the DEBSIDE trial
}

\author{
Jacques Berland $^{1 *}$, MD; Thierry Lefèvre ${ }^{2}$, MD, FESC, FSCAI; Philippe Brenot ${ }^{3}$, MD; Jean Fajadet ${ }^{4}$, MD; \\ Pascal Motreff ${ }^{5}, \mathrm{MD}, \mathrm{PhD}$; Patrice Guerin ${ }^{6}, \mathrm{MD}$, PhD; Patrick Dupouy ${ }^{7}, \mathrm{MD}$; Christian Schandrin ${ }^{8}, \mathrm{MD}$; \\ DEBSIDE trial investigators
}

1. Clinique Saint-Hilaire, Rouen, France; 2. Institut Hospitalier Jacques Cartier, Massy, France; 3. Centre Cardiologique d'Evecquemont, Evecquemont, France; 4. Clinique Pasteur, Toulouse, France; 5. Hôpital Gabriel Montpied, Clermont-Ferrand, France; 6. Hôpital Guillaume et René Laennec, Nantes, France; 7. Clinique les Fontaines, Melun, France; 8. Pôle Santé République, Clermont-Ferrand, France

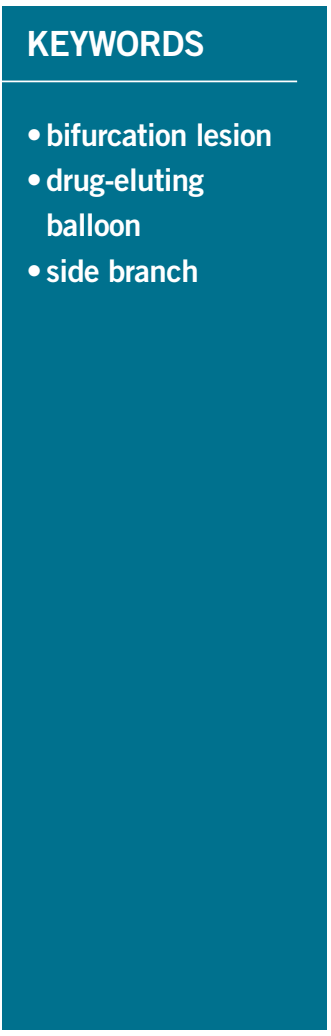

\begin{abstract}
Aims: We aimed to evaluate the role of drug-eluting balloon SB inflation, using the novel DANUBIO balloon, after placement of a drug-eluting stent in the main branch in patients with bifurcation lesions.

Methods and results: Fifty-two patients with bifurcation lesions suitable for stenting were enrolled in the DEBSIDE trial at eight French centres between May 2012 and July 2013. Two patients were excluded from the trial because of significant protocol deviations. Systematic Nile PAX stent placement was followed by final drug-eluting balloon inflation, using the DANUBIO balloon, according to the size of the side branch. Clinical follow-up was scheduled at one, six, and twelve months and an angiographic control at six months. The primary endpoint was six-month late lumen loss (LLL) at the ostium of the side branch. Secondary endpoints were main branch (MB) LLL, binary restenosis of the SB and MB, and clinically driven revascularisation rates for both branches. The procedural success rate was $100 \%$. Angiographic control at six months post-procedure was performed in 48 patients (96\%). Two patients with no reported clinical events refused the angiographic control. At six-month follow-up the primary endpoint of side branch LLL was $-0.04 \pm 0.34 \mathrm{~mm}$ and the secondary endpoint of MB LLL was $0.54 \pm 0.60 \mathrm{~mm}$. There was only one myocardial infarction (2\%) and no reported cardiac deaths. Only one patient (2\%) had a non-clinically driven target lesion revascularisation (TLR) at the level of the side branch combined with a main branch revascularisation.
\end{abstract}

Conclusions: Systematic final inflation of a DANUBIO balloon in the side branch after placement of a Nile PAX stent in the main branch for the treatment of a bifurcation lesion is safe and effective and results in very low LLL and a low restenosis rate at the side branch ostium. The DEBSIDE clinical trial was registered at the United States National Institute of Health website (NCT01485081).

\footnotetext{
*Corresponding author: Cardiology Department, Clinique Saint-Hilaire, 2 place Saint-Hilaire, 76000 Rouen, France. E-mail: jberland@clinique-sainthilaire.fr
} 


\section{Abbreviations}

$\begin{array}{ll}\text { BTHC } & \text { butyryl tri-n-hexyl citrate } \\ \text { CEC } & \text { clinical events committee } \\ \text { DEB } & \text { drug-eluting balloon } \\ \text { DES } & \text { drug-eluting stent } \\ \text { DM } & \text { distal main branch } \\ \text { DS } & \text { diameter stenosis } \\ \text { LLL } & \text { late lumen loss } \\ \text { MACE } & \text { major adverse cardiac events } \\ \text { MB } & \text { main branch } \\ \text { MBC } & \text { main branch catheter } \\ \text { MI } & \text { myocardial infarction } \\ \text { MLD } & \text { minimum lumen diameter } \\ \text { PCI } & \text { percutaneous coronary intervention } \\ \text { PM } & \text { proximal main branch } \\ \text { POBA } & \text { plain old balloon angioplasty } \\ \text { QCA } & \text { quantitative coronary angiography } \\ \text { RVD } & \text { reference vessel diameter } \\ \text { SB } & \text { side branch } \\ \text { SBC } & \text { side branch catheter } \\ \text { TLR } & \text { target lesion revascularisation } \\ \text { TVR } & \text { target vessel revascularisation } \\ & \end{array}$

\section{Introduction}

Coronary bifurcation lesions continue to be considered complex coronary lesions to treat. The introduction of drug-eluting stents (DES) has lowered the rate of restenosis in these lesions ${ }^{1}$. Even so, in the recent consensus document of the European Bifurcation Club, the complexity of treatment was again underlined, calling it "one of the most challenging lesions in interventional cardiology in terms of procedural success rate as well as long-term cardiac events", and the optimal treatment of the side branch (SB) in a coronary bifurcation lesion remains a matter of considerable debate within the current literature $^{3,4}$. Even if several studies have been published on the role of drug-eluting balloons (DEB) in the main branch (MB) and/or SB in combination with a bare metal stent (BMS) in the $\mathrm{MB}^{5-9}$, to date only one published study has evaluated the role of DEB inflation in the $\mathrm{SB}$ after placement of a DES in the $\mathrm{MB}^{10}$, with promising results.

A previous study, the BIPAX trial ${ }^{11}$, evaluated a dedicated paclitaxel-eluting stent (Nile PAX ${ }^{\circledR}$; Minvasys, Gennevilliers, France) for the treatment of coronary bifurcation lesions in 101 patients. The nine-month angiographic results showed an $18.4 \%$ rate of restenosis, including $12.8 \%$ at the ostium of the side branch.

With this in mind, we investigated a new drug-eluting balloon, DANUBIO (Minvasys), for the treatment of side branches after the systematic implantation of the Nile PAX dedicated drug-eluting stent in the main branch. The efficacy of the DANUBIO was assessed by means of an angiographic control performed in all patients.

\section{Methods STUDY DESIGN}

The DEBSIDE clinical trial was a prospective, non-randomised, multicentre, interventional study evaluating the investigational
DANUBIO paclitaxel-eluting coronary balloon for the treatment of side branches of de novo bifurcation lesions with a side branch reference vessel diameter $\geq 2.0 \mathrm{~mm}$ and $\leq 3.0 \mathrm{~mm}$.

\section{STUDY DEVICE}

The DANUBIO paclitaxel-eluting PTCA balloon catheter (Minvasys) is a new technology developed in order to treat coronary artery disease and especially restenosis. The balloon surface is coated with $2.5 \mu \mathrm{g} / \mathrm{mm}^{2}$ paclitaxel incorporated in a proprietary delivery matrix of butyryl tri-n-hexyl citrate (BTHC). The DANUBIO device has been thoroughly evaluated in two preclinical studies with animal investigations. An efficacy study involved an assessment of acute drug release into the vessel wall, pharmacokinetics of the BTHC/paclitaxel formulation as well as the residual drug on the balloon catheter. Device safety was evaluated in a prospective randomised study performed in 20 porcine coronary arteries at IMM Research, Paris, France. The data were assessed through control angiography, examination and explantation of the heart followed by histopathologic and histomorphometric analysis of the treated arteries at 28 days after procedure. The control device was a bare balloon catheter and demonstrated a higher mean restenosis than the DEB group. The results showed device safety with no clinical cardiac events, no sign of toxicity, no fibrin deposits or thrombi in the lumen of the treated artery (data on file). Further to these preclinical trial results, the DANUBIO obtained CE mark approval in October 2011.

The DANUBIO study device was used in conjunction with the Nile PAX, a polymer-free abluminal-coated paclitaxel-eluting coronary stent dedicated to bifurcation lesions. The stent platform is an L605 cobalt-chromium ( $\mathrm{Co}-\mathrm{Cr}$ ) stent and is designed with a single link at the level of the carina to prevent side branch obstruction. The delivery system is $6 \mathrm{Fr}$ compatible and is composed of two parallel rapid exchange (RX) balloon catheters, the main branch catheter (MBC) and side branch catheter (SBC), which can be used in a final kissing post-dilatation to optimise deployment. The Nile PAX allows an optimal provisional T-stenting approach. The device has been evaluated in the BIPAX triall $^{11}$ (NCT01308229).

\section{PATIENT POPULATION}

Between May 2012 and July 2013, 52 patients with de novo bifurcation lesions (except Medina class 0,0,1 type lesions) were treated with the DANUBIO drug-eluting balloon in eight clinical centres in France. Inclusion criteria for enrolment in the trial included male and non-pregnant female patients over 18 years of age with clinical evidence of ischaemic heart disease, stable or unstable angina with signs of ischaemia, silent ischaemia, or a positive functional test. Lesion length had to be $\leq 6 \mathrm{~mm}$ in the side branch and the reference vessel diameter had to be between 2.5 and $3.5 \mathrm{~mm}$ for the $\mathrm{MB}$ and 2.0 and $3.0 \mathrm{~mm}$ for the $\mathrm{SB}$. Exclusion criteria included previous percutaneous coronary intervention (PCI) of the target vessel within six months prior to the procedure, left main bifurcation and acute myocardial 
infarction within 72 hours. Two patients were excluded postprocedure because of significant protocol deviations: one patient had a previous PCI at the target lesion and the other had a side branch lesion longer than $10 \mathrm{~mm}$ (Figure 1).

All patients provided written informed consent prior to enrolment and procedure. The study complied with the Declaration of Helsinki regarding investigation in humans, and was approved by the local ethics committee.

\section{PROCEDURE}

First, a sequential main branch and side branch predilatation was performed with a conventional balloon. If the angiographic result was good after predilatation, a dedicated drug-eluting stent (Nile PAX) was deployed in the main branch of the bifurcation site. The stent diameter was selected based on the diameter of the distal MB. A recommended kissing balloon procedure was then performed with the MBC and SBC of the Nile PAX delivery system. After that, the guidewire in the main branch was removed in order to prevent any drug loss when the DEB was placed into the side branch. Indeed, as already reported by Afari et $\mathrm{al}^{12}$, the active coating is subject to erosion during tracking to the target lesion, notably in the guiding catheter. The use of a second wire is a potential cause of increased friction resulting in loss of coating.

If there was no dissection impeding the flow in the side branch, then the DEB (DANUBIO balloon catheter) was inflated only once in the side branch for 30 seconds at low pressure (8-10 bar). The procedure was performed based on the recommendations of the German consensus group ${ }^{13}$. As defined in the protocol, the DEB diameter could not exceed in any way the SB diameter and had to match the Nile PAX SBC diameter. In addition, the balloon length had to extend at least $2 \mathrm{~mm}$ beyond the side branch lesion distally in order to avoid geographical mismatch.

Patients then received dual antiplatelet therapy (DAPT) for at least 12 months.

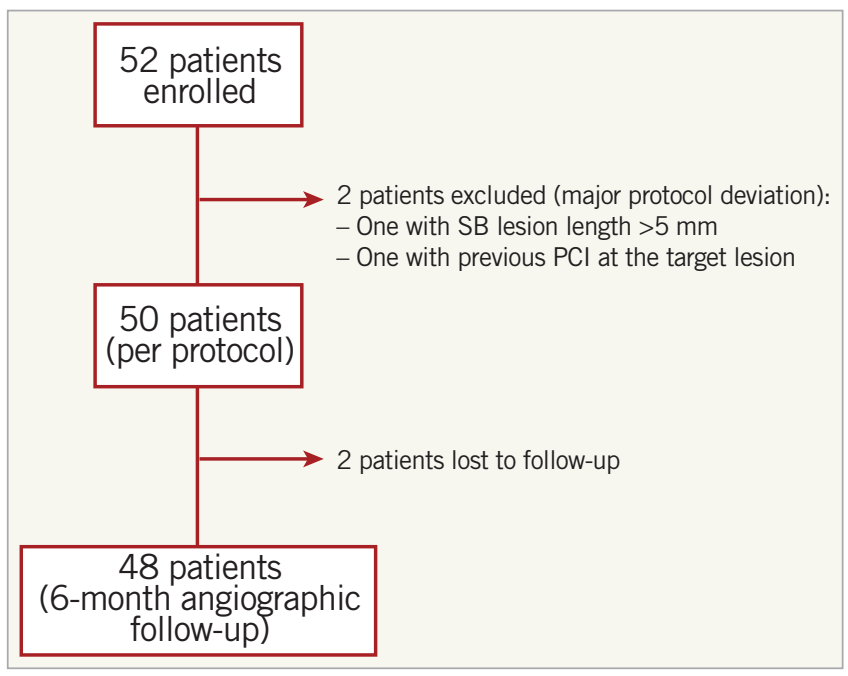

Figure 1. Patient CONSORT-type flow chart.

\section{ANGIOGRAPHIC ANALYSIS}

Serial coronary angiographic studies were obtained at baseline (pre-procedure) and post-procedure after intracoronary administration of nitrate (recommended 100-200 $\mu \mathrm{g}$, unless clinically contraindicated) in two orthogonal matching views. For the study, the dedicated bifurcation algorithm from the CAAS 5.10 system (Pie Medical Imaging BV, Maastricht, The Netherlands) was used. All angiographic analyses were performed by an independent angiographic core laboratory (European Cardiovascular Research Center [CERC], Massy, France). A single analysis was performed after placing boundaries for the proximal and distal main branch and side branch segments. The worst analysable view was selected. Automatic contour detection was used; manual editing was restricted to cases where the contour detection was not following the vessel. Both main and side branch reference diameters were automatically interpolated by the software using the automatic segmentation. Both six-segment and 11-segment models were analysed; however, due to the limitations related to the role of the point of convergence (segment 7) in the 11-segment models, data are presented according to the six-segment model as proposed by Ramcharitar et $\mathrm{al}^{14}$ and Girasis et $\mathrm{al}^{15}$. The minimum lumen diameter (MLD) of the side branch was evaluated at the level of the ostium in the first $5 \mathrm{~mm}$ of the proximal portion of the side branch. The late lumen loss (LLL) was calculated as usual: $\mathrm{LLL}=\mathrm{MLD}$ post-procedure - MLD at six months.

\section{STUDY ENDPOINTS}

The primary endpoint of the study was the evaluation of LLL at the ostium of the side branch at six months by quantitative coronary angiography (QCA) analysis. Secondary endpoints were angiographic success, LLL in the main branch, angiographic binary restenosis $(\mathrm{ABR})$ rate $(\%)$ in the side and main branches, and major adverse cardiac events (MACE: cardiac death, myocardial infarction $[\mathrm{MI}]$ and target lesion revascularisation [TLR] in the MB and SB). The procedure success was also measured (success defined as achieving a stenosis of less than $50 \%$ with no clinical or ischaemic events during the initial hospitalisation). Clinical follow-up was scheduled at one, six and twelve months, and patients had systematic six-month angiographic analysis. A clinical events committee (CEC) adjudicated all clinical events.

\section{STATISTICAL ANALYSIS}

Statistical analysis was performed by an independent core laboratory (CERC). All patients/lesions were analysed on a per protocol basis. The continuous variables are described as mean \pm standard deviation, and discrete variables with frequencies and percentages. All analyses were carried out using SAS software, version 9.4 (SAS Institute Inc., Cary, NC, USA).

The Student's t-test was used for the comparisons analysis between results obtained before and after the procedure and at six-month follow-up. A p-value $<0.05$ was considered statistically significant. 


\section{Results}

\section{BASELINE CHARACTERISTICS}

Patients' clinical demographics are presented in Table 1.

The clinical profile of the 50 patients included in the protocol analysis was typical of a population suffering from coronary artery disease with an average age of 66 years, $78 \%$ male, $26 \%$ rate of diabetes and a previous history of ischaemic heart disease in $40 \%$ of patients. Fifty percent of patients were clinically stable, either with stable angina or with silent ischaemia, whereas $50 \%$ of patients were unstable, with $28 \%$ STEMI or NSTEMI. The most frequently treated bifurcation involved the LAD and a diagonal artery (diagonal 1 in 30 patients [ $60 \%$ ], diagonal 2 in five patients [10\%], followed by marginal circumflex artery 1 and 2 in nine patients [18\%], and the right posterior lateral artery/right descending posterior artery (RPL/RDP) bifurcation in the right coronary artery (RCA) in four patients [8\%]).

The bifurcation lesions, described according to the Medina classification based on visual estimate by the investigators (Figure 2),

Table 1. Baseline clinical demographics.

\begin{tabular}{|l|c|}
\multicolumn{1}{|c|}{ Variable } & $\mathbf{n = 5 0}$ \\
\hline Age, years & $65.8 \pm 9.6$ \\
\hline Male, \% & 78.0 \\
\hline Active smoker, \% & 16.0 \\
\hline Hypertension, \% & 76.0 \\
\hline Hypercholesterolaemia,\% & 54.0 \\
\hline Diabetes, \% & 26.0 \\
\hline History of CAD, \% & 40.0 \\
\hline History of MI $>28$ days & 8.0 \\
\hline History of stroke & 2.0 \\
\hline DES implantation & 22.0 \\
\hline BMS implantation & 8.0 \\
\hline Previous CABG & 2.0 \\
\hline Clinical presentation, \% & \\
\hline Stable angina & 36.0 \\
\hline Unstable angina & 20.0 \\
\hline Silent ischaemia & 14.0 \\
\hline STEMI ST-/ST+ & 28.0 \\
\hline
\end{tabular}

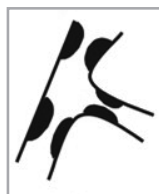

$1,1,1$

$16(32.0 \%)$

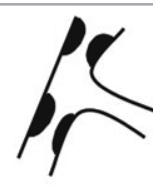

$1,1,0$

$12(24.0 \%)$

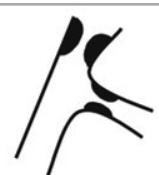

$1,0,1$

$13(26.0 \%)$

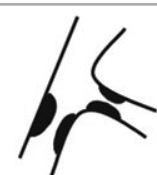

$0,1,1$

$3(6.0 \%)$

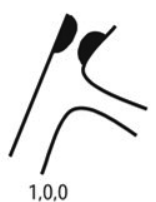

$5(10.0 \%)$

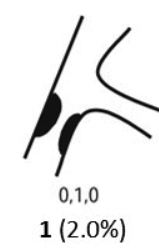

$(2.0 \%)$

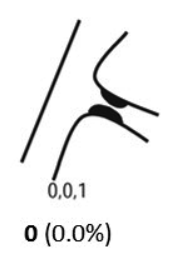

Figure 2. Medina classification of lesions. comprised 16 type 1,1,1 lesions, 13 type 1,0,1 lesions, and three type $0,1,1$ lesions.

In total, 32 patients $(64 \%)$ presented with a visually significant lesion of the side branch. For the 18 other patients, the distribution of lesions was as follows: 12 type 1,1,0 lesions, five type 1,0,0 lesions, and one type 0,1,0 lesion.

\section{PROCEDURAL CHARACTERISTICS}

The main branch was predilated in 50 patients and the side branch in 41 patients. All patients with good angiographic results were then treated with the Nile PAX drug-eluting stent in the main branch.

The distal diameter of the dedicated stent used was $3.5 \mathrm{~mm}$ for 10 patients, $3.0 \mathrm{~mm}$ for 33 patients and $2.50 \mathrm{~mm}$ for seven patients. Then, a kissing inflation with the balloon dedicated to the side branch of the Nile PAX system was performed on 40 patients (diameter $2.0 \mathrm{~mm}$ for six patients, $2.5 \mathrm{~mm}$ for 26 patients and $3.0 \mathrm{~mm}$ for eight patients). For the remaining patients, the opening of the SB post-stenting was performed with SB inflation only.

After checking that there was no dissection of the ostium of the side branch and after removal of the guidewire in the main branch to avoid any friction between the drug-eluting balloon and guidewire, the DANUBIO balloon was successfully inflated in the side branch of all 50 patients for a mean duration of a $41 \pm 25$ seconds. The diameter of the DANUBIO balloon was chosen by the investigator according to visual estimation: a $2 \mathrm{~mm}$ balloon was chosen for 11 patients, a $2.5 \mathrm{~mm}$ balloon for 26 patients and a $3 \mathrm{~mm}$ balloon for 13 patients. The mean balloon inflation pressure was 9.4 \pm 2.2 atmospheres. One Amazonia PAX (Minvasys) stent was positioned distally in the main branch for a residual dissection.

In total, taking this additional stent into account, the procedure was successful in $100 \%$ of cases.

\section{ANGIOGRAPHIC RESULTS}

Angiographic analysis was possible for all patients before and immediately after the procedure, whereas angiographic follow-up at six months was performed on 48 patients (96\%). The results of this analysis are given in Table 2.

Before the procedure, the reference diameter of the proximal main branch was $2.93 \pm 0.43 \mathrm{~mm}$, which did not change after the procedure $(3.03 \pm 0.38 \mathrm{~mm})$. The reference diameter of the side branch was $1.84 \pm 0.34 \mathrm{~mm}$, without modification after the procedure. Mean lesion length was $12.0 \pm 3.5 \mathrm{~mm}$ in the main branch and $5.50 \pm 0.97 \mathrm{~mm}$ in the secondary branch. The percentage stenosis in the main branch before the procedure was $62 \pm 14 \%$, decreasing to $26 \pm 11 \%$ after placement of the stent. The mean percentage of the side branch diameter stenosis was $30.0 \pm 14.5 \%$ before the procedure, decreasing to $23.9 \pm 11.3 \%$ after the procedure.

Representations of the minimum lumen diameter (MLD) in the side branch including cumulative frequency curves for all patients pre-procedure, post-procedure and at follow-up are presented in Figure $3 \mathbf{A}$ and Figure 3B.

The MLD of the side branch increased from $1.36 \pm 0.38 \mathrm{~mm}$ to $1.52 \pm 0.31 \mathrm{~mm}$ after the procedure $(\mathrm{p}=0.01)$, and remained 
Table 2. Angiographic acute and 6-month follow-up results.

\begin{tabular}{|c|c|c|c|}
\hline & MB proximal & MB distal & SB \\
\hline \multicolumn{4}{|l|}{ Pre-procedure } \\
\hline $\mathrm{RVD}, \mathrm{mm}$ & $2.93 \pm 0.43$ & $2.16 \pm 0.39$ & $1.84 \pm 0.34$ \\
\hline MLD, mm & $1.39 \pm 0.62$ & $1.44 \pm 0.50$ & $1.36 \pm 0.38$ \\
\hline DS,\% & $52.6 \pm 20.0$ & $33.3 \pm 19.5$ & $29.8 \pm 14.5$ \\
\hline \multicolumn{4}{|l|}{ Post-procedure } \\
\hline $\mathrm{RVD}, \mathrm{mm}$ & $3.03 \pm 0.38$ & $2.48 \pm 0.32$ & $1.83 \pm 0.33$ \\
\hline MLD, mm & $2.62 \pm 0.31$ & $2.18 \pm 0.33$ & $1.52 \pm 0.31$ \\
\hline $\mathrm{DS}, \%$ & $13.1 \pm 6.5$ & $11.9 \pm 6.7$ & $23.9 \pm 11.3$ \\
\hline Acute gain & $1.18 \pm 0.68$ & $0.74 \pm 0.45$ & $0.18 \pm 0.34$ \\
\hline \multicolumn{4}{|l|}{ 6-month follow-up } \\
\hline Angiographic binary restenosis,\% & 4.0 & 14.0 & 2.0 \\
\hline $\mathrm{RVD}, \mathrm{mm}$ & $2.91 \pm 0.47$ & $2.22 \pm 0.42$ & $1.96 \pm 0.34$ \\
\hline MLD, mm & $2.02 \pm 0.54$ & $1.52 \pm 0.50$ & $1.55 \pm 0.35$ \\
\hline $\mathrm{DS}, \%$ & $30.1 \pm 16.5$ & $31.9 \pm 18.8$ & $25.7 \pm 12.6$ \\
\hline In-stent LLL, mm & $0.61 \pm 0.53$ & $0.69 \pm 0.46$ & $-0.04 \pm 0.34$ \\
\hline MB in-stent LLL, mm & \multicolumn{2}{|c|}{$0.54 \pm 0.60$} & - \\
\hline
\end{tabular}

identical at $1.55 \pm 0.35 \mathrm{~mm}$ at six months. The LLL at the ostium was, on average, slightly negative $(-0.04 \pm 0.34 \mathrm{~mm})$ and homogenous in the population, with no difference between the group of patients with significant lesions of the side branch before the procedure $(n=32)$ and the group without side branch lesions $(n=18)$. The MLD of the proximal and distal main branch provided in Table 2 increased from $1.39 \pm 0.62 \mathrm{~mm}$ and $1.44 \pm 0.50 \mathrm{~mm}$ before the procedure to $2.62 \pm 0.31 \mathrm{~mm}$ and $2.18 \pm 0.33 \mathrm{~mm}$ after the procedure

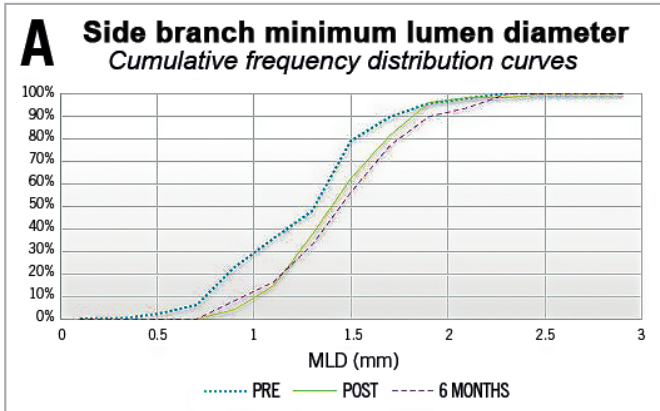

B Side branch minimum lumen diameter

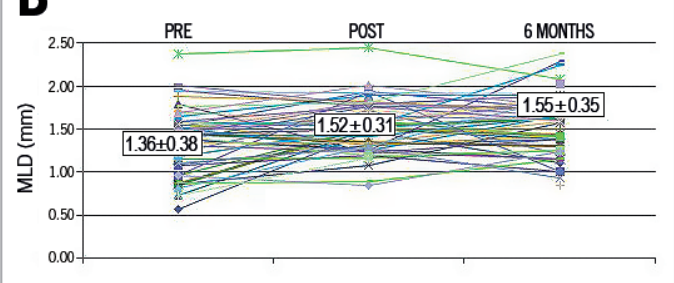

Figure 3. Minimum lumen diameters of the side branch: preprocedure, post-procedure and at follow-up. A) Cumulative frequency distribution curves. B) Individual data.
( $\mathrm{p}<0.0001$ ), respectively, and it decreased to $2.02 \pm 0.54 \mathrm{~mm}$ and $1.52 \pm 0.50 \mathrm{~mm}$ at six months $(\mathrm{p}<0.0001)$.

Note that the reference diameter of the side branch increased between post-procedure and six months from $1.83 \pm 0.33 \mathrm{~mm}$ to $1.96 \pm 0.34 \mathrm{~mm}(\mathrm{p}=0.06)$. A graphical representation of the location of the different restenoses is provided in Figure 4. Six were located in the distal portion of the main branch stent, one at the bifurcation segment of the stent matched with the only ostial side branch restenosis, one in the proximal segment of the stent and two at the edges.

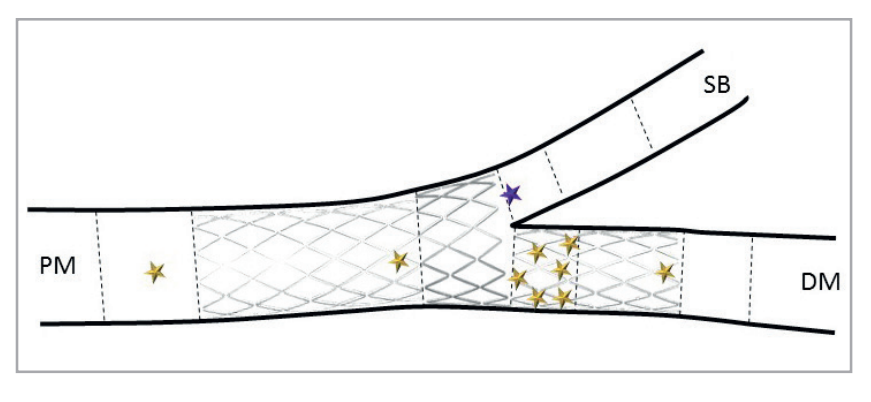

Figure 4. Restenosis localisation. DM: distal main branch; PM: proximal main branch; $S B$ : side branch

\section{CLINICAL OUTCOME}

There were no in-hospital complications and no adverse clinical events up to 30 days, except for a single myocardial infarction (MI: 2.0\%) where the patient received emergency revascularisation for an acute occlusion in the target lesion at the level of the stent. There were no additional MI and, within the six-month follow-up, only one patient had a non-clinically driven TLR at the level of the side branch combined with a main branch revascularisation $(2.0 \%)$. Non-clinically driven re-dilation was performed on three patients $(6.0 \%)$ at the level of the lesion in the main vessel, one patient on the vessel beyond the lesion, and in two patients angioplasty was performed on another vessel at six months. A total of five major adverse cardiac events (hierarchical and cumulative: $10.0 \%$ ) were noted during follow-up. To date, there has been no reported cardiac death $(0.0 \%)$.

\section{Discussion}

The main results of the DEBSIDE study were the good angiographic and clinical outcomes in the side branch after treatment with a drug-eluting balloon at six months post-procedure. Although the angiographic results obtained in the main branch were not as favourable as in the side branch, the clinical results were acceptable. Indeed, only $6.0 \%$ of patients needed repeat revascularisation at the target lesion at the level of the main branch.

The treatment of bifurcated coronary artery stenosis remains challenging and the complication rate is high. PCI in bifurcation lesions is associated with lower immediate angiographic and clinical success and higher rates of restenosis, which, in addition, are more challenging to treat compared to non-bifurcated lesions ${ }^{16}$. 
Data from the Nordic Bifurcation Study ${ }^{16}$ and the British Bifurcation Coronary study ${ }^{17}$ (BBC ONE) showed that the method of choice to treat bifurcation lesions is stenting the main branch and only dilating the side branch, because double stenting appears not to lower the risk of restenosis, while the placement of two stents in the bifurcation makes the procedure more difficult to perform. Thus, the current practice to treat bifurcation lesions is the provisional T-stenting technique, i.e., stenting only the $\mathrm{MB}$ and final kissing balloon, avoiding SB stenting when possible. However, with this technique, whilst the success rate in the main branch is satisfactory, the rate of restenosis in the side branch remains problematic. At the six-month angiographic follow-up in Nordic Bifurcation I, the restenosis rate in the side branch was $19.2 \%$ when the side branch was treated with a simple strategy without placement of a second stent, and $11.5 \%$ when a second drug-eluting stent was positioned in the side branch ${ }^{16}$.

In the BBC ONE clinical study ${ }^{17}$, where there was no systematic angiographic follow-up, the restenosis rate in the side branch was $2.7 \%$ in the simple strategy group.

The most recent study with angiographic follow-up is the DKCRUSH-II study ${ }^{18}$. In this study, which used a complex double kissing technique with two stents, the late lumen loss in the side branch was assessed as $0.22 \pm 0.41 \mathrm{~mm}$ and it was identical in the control group with one drug-eluting stent in the main branch and a conventional balloon in the secondary branch $(0.18 \pm 0.45 \mathrm{~mm})$. All of these publications confirm that the result of treatment of the side branch remains problematic since the use of a drug-eluting stent as compared to a conventional balloon does not seem to yield significant improvements.

For this reason, the use of a drug-eluting balloon may be useful to provide an antiproliferative substance to a lesion without the use of a polymer-covered stent.

Over the past few years, drug-eluting balloons have demonstrated their clinical safety and efficacy for the treatment of small coronary vessels ${ }^{19}$ and in-stent restenosis ${ }^{20,21}$. Drug-eluting balloons have now received a guideline ${ }^{22}$ class I level of evidence A recommendation in the treatment of in-stent restenosis after bare metal stent (BMS) or drug-eluting stent implantation.

To date, there have been very few clinical trials on the treatment of bifurcation lesions with drug-eluting balloons and all of these included only a small cohort of patients.

The DEBIUT registry ${ }^{5}$ enrolled 20 patients with coronary artery bifurcation lesions. A DEB (the first generation of the DIOR $^{\circledR}$ balloon catheter; Eurocor GmbH, Bonn, Germany) was used during the predilatation step in both main and side branches before provisional main branch stenting with a BMS. Final kissing inflation with regular balloons was performed according to routine practice. The procedure was successful in all patients. The use of sequential predilatation with a DEB was considered safe and well tolerated. No stents were placed in the SB. No MACE or reintervention occurred during the four-month clinical follow-up period, and there was no subacute vessel closure. This registry provided encouraging results for the use of a DEB in bifurcation treatment to improve long-term side branch patency. However, the larger randomised DEBIUT study ${ }^{6,7}$, initiated after the registry, did not confirm these initial promising results. Indeed, the outcome of the primary endpoint was not reached. The proximal $\mathrm{MB}$ late lumen loss was better in the group treated with paclitaxel DES+plain old balloon angioplasty (POBA) $(0.18 \mathrm{~mm})$ than in the group treated with sequential MB/ $\mathrm{SB}$ dilatation with the DEB then BMS implantation in the MB $(0.42 \mathrm{~mm})$. Similarly, the SB LLL was better in the DES+POBA group (DEB+BMS $0.19 \pm 0.66 \mathrm{~mm}$ vs. DES $0.11 \pm 0.43$ ). These "poor" results obtained with the DIOR I led to the development of the DIOR II (second generation) with a coating involving a new excipient (shellac) ${ }^{7}$. Preclinical results obtained with the DIOR II showed much higher drug concentrations into the vessel wall than the DIOR ${ }^{23}$. This emphasises the importance of the excipient in DEB as discussed by Afari et $\mathrm{al}^{12}$.

The PEPCAD V trial ${ }^{8}$ was a feasibility and safety first-in-man series evaluating a DEB (SeQuent ${ }^{\circledR}$ Please balloon catheter; B. Braun Melsungen AG, Melsungen, Germany) in the treatment of significant de novo bifurcation stenosis. The MB and SB were predilated with the DEB followed by a bare metal stent (Coroflex ${ }^{\circledR}$ stent; B. Braun Melsungen AG) deployment in the main branch. Twenty-eight patients were included in the study. There were no MACE up to 30 days and no TLR was performed. However, two late stent thromboses were reported (one definite and one probable at six and eight months after the index procedure, respectively). Nine-month angiographic results showed an in-stent late lumen loss of $0.38 \pm 0.46 \mathrm{~mm}$ in the main branch and an in-lesion unsatisfactory late lumen loss of $0.21 \pm 0.48 \mathrm{~mm}$ in the side branch.

In a more recent prospective randomised clinical study, the BABILON trial ${ }^{9}$, sequential $\mathrm{MB} / \mathrm{SB}$ dilatation with a DEB (SeQuent Please), with provisional T-stenting with a BMS in the MB (52 patients), was compared to a sequential MB/SB dilatation with conventional plain balloons followed by everolimus DES implantation in the MB (56 patients). As opposed to the previous study, both strategies showed similar good results in the SB, LLL of $-0.04 \pm 0.71 \mathrm{~mm}$ in the DEB followed by BMS group, and $-0.03 \pm 0.51 \mathrm{~mm}$ in the DEB followed by everolimus stent group.

In the literature, only one clinical trial has studied the combination of DES in the MB followed by DEB in the SB. Indeed, in a recent study conducted by Herrador et $\mathrm{al}^{10}$, a DES (TAXUS ${ }^{\mathrm{TM}}$ LibertéTM; Boston Scientific, Marlborough, MA, USA) was implanted in the main branch, which is the usual approach for the treatment of a bifurcation lesion, and the SB was treated with a DEB (SeQuent Please). Fifty patients treated with the DEB were compared to 50 patients treated with a conventional balloon in the SB. Angiographic follow-up and IVUS were scheduled at 12 months post-procedure. At angiographic follow-up, there was a lower percentage of SB restenosis in the DEB group ( $20 \%$ vs. $7 \%, p=0.08)$, with less SB late loss $(0.40 \pm 0.50 \mathrm{~mm}$ vs. $0.09 \pm 0.40 \mathrm{~mm}, \mathrm{p}=0.01)$ and similar MB late loss $(0.62 \pm 0.70 \mathrm{~mm}$ vs. $0.49 \pm 0.60 \mathrm{~mm}, \mathrm{p}=0.39$ ). The MACE rate at 12-month followup was $24 \%$ in the group treated by a conventional balloon versus 
$11 \%$ in the DEB group $(\mathrm{p}=0.11)$. The TLR rate was also higher in the conventional balloon group ( $22 \%$ vs. $12 \%, p=0.16)$.

The angiographic and clinical results obtained in our study are highly encouraging for the use of DEB as the final treatment in the side branch and are significantly better than the results obtained with the same stent in the BIPAX study involving the same stenting strategy without using a DEB in the SB (-0.04 vs. $0.33 \mathrm{~mm}$ LLL, respectively). Indeed, in the present study, only one single patient had restenosis and target lesion revascularisation at the level of the side branch. The association of the discrete increase in the reference diameter of the side branch and the unchanged MLD in the ostium of the side branch explains the very low restenosis rate (defined as a stenosis greater than $50 \%$ diameter) of $2 \%$ in the side branch, whereas it reached $4.0 \%$ in the proximal $\mathrm{MB}$ and $14.0 \%$ in the distal MB. The side branch late lumen loss measured six months after the index procedure $(-0.04 \pm 0.34 \mathrm{~mm})$ was similar to BABILON trial results ${ }^{9}$, slightly better than Herrador's study ${ }^{10}$ or much better than that in previously published studies on bifurcation treatment with DEB (Table 3) or that obtained with DES ${ }^{16-24}$.

This good result may be explained by the fact that inflation of the drug-eluting balloon was performed at the end of the procedure, after opening of the stent strut into the side branch facilitated by the design of the dedicated Nile PAX stent, without further inflation (as observed in some other protocols), and also because removal of the guidewire in the main branch to avoid friction and any potential loss of the drug was recommended. Although less convenient than a dedicated stent, in case the stent used in combination with the DEB is a tubular stent, the opening of the stent strut into the side branch is considered mandatory before implementing the DEB. Crossing the stent struts with the DEB is therefore not an issue.

Finally, the main reason for restenosis in areas where no stent is implanted is elastic recoil that was often observed after

Table 3. Side branch late lumen loss at six months in DEBSIDE and previous trials.

\begin{tabular}{|l|l|l|c|c|}
\hline \multicolumn{1}{|c|}{ Trial } & \multicolumn{1}{|c|}{$\begin{array}{c}\text { Study } \\
\text { device }\end{array}$} & \multicolumn{1}{|c|}{ Technique } & Months & SB LLL (mm) \\
\hline $\begin{array}{l}\text { DEBSIDE } \\
(2014)\end{array}$ & DANUBI0 & $\begin{array}{l}\text { Predilatation: conventional } \\
\text { balloon in MB+SB } \\
\text { MB: DES } \\
\text { SB: DEB }\end{array}$ & 6 & $-0.04 \pm 0.34$ \\
\hline $\begin{array}{l}\text { PEPCAD V } \\
(2011)^{8}\end{array}$ & $\begin{array}{l}\text { SeQuent } \\
\text { Please }\end{array}$ & $\begin{array}{l}\text { MB+SB: DEB } \\
\text { MB: BMS }\end{array}$ & 9 & $0.21 \pm 0.47$ \\
\hline $\begin{array}{l}\text { DEBIUT } \\
(2011)^{7}\end{array}$ & DIOR I & $\begin{array}{l}\text { Predilatation: DEB in } \\
\text { MB+SB } \\
\text { MB: BMS }\end{array}$ & 6 & $0.19 \pm 0.66$ \\
\hline $\begin{array}{l}\text { Herrador } \\
\text { et al } \\
(2013)^{10}\end{array}$ & $\begin{array}{l}\text { SeQuent } \\
\text { Please }\end{array}$ & $\begin{array}{l}\text { Predilatation: conventional } \\
\text { balloon in MB or SB } \\
\text { SB: DEB } \\
\text { MB: DES }\end{array}$ & 12 & $0.09 \pm 0.40$ \\
\hline $\begin{array}{l}\text { BABILON } \\
(2014)^{9}\end{array}$ & $\begin{array}{l}\text { SeQuent } \\
\text { Please }\end{array}$ & $\begin{array}{l}\text { Predilatation: conventional } \\
\text { balloon in MB+SB } \\
\text { MB+SB: DEB } \\
\text { MB: BMS }\end{array}$ & 9 & $-0.04 \pm 0.64$ \\
\hline
\end{tabular}

conventional balloon angioplasty ${ }^{25-27}$. In this study, the absence of restenosis in the SB combined with dilatation of the side branch reference diameter is also an argument in favour of elimination of elastic recoil after the use of a drug-eluting balloon, as has already been noted in a study on DEB in the small vessels ${ }^{19}$.

Device safety was indicated by a low MACE rate up to six months $(10.0 \%)$. Most of the clinical events were induced by the need for target lesion revascularisation at the level of the main branch $(6.0 \%)$. The rate of TLR at the level of the MB does not seem to be linked to a distortion of the stent due to an absence of final kissing, as for the majority of patients a kissing inflation was performed post-stenting before the inflation of the DEB to ensure a proper stent apposition. The DEB was inflated afterwards at a low pressure with the same diameter as the side branch catheter of the dedicated stent, limiting the risk of further stent distortion. An overdosage of paclitaxel does not seem to be a potential cause as there were no visual aneurysms and there was a single case of restenosis located at the level of the overlap between the DEB and the DES. The same trend of a relatively high TLR rate and restenosis in the $\mathrm{MB}$ was noticed in the BIPAX study, where no DEB was involved. Similar results were also observed in the BABILON trial ${ }^{9}$ where higher LLL and incidence of MACE in the MB were observed compared to SB. Moreover, although high, our restenosis rate in the MB $(18 \%)$ was similar to the results obtained with the first-generation DES (TAXUS) in a large series of patients with complex lesions and systematic follow-up angiography $(18.9 \%)^{28}$.

Therefore, the hypothetical reason for these relatively high TLR and restenosis rates at the level of the MB is the antiproliferative drug used on the stent (paclitaxel) and the coating formulation without polymer.

In view of these results, a new sirolimus-eluting biodegradable polymer stent (Nile SIR) is currently being developed by the sponsor.

\section{Limitations}

A certain number of limitations should be noted. The study was a first-in-man non-randomised trial with no control group. The study included a relatively low number of patients (50; no calculation was used to determine sample size), and only 32 of these had a significant lesion on both the main vessel and the side branch. Indeed, it was a relatively healthy SB population with, therefore, a low acute gain which however remained unchanged six months post-procedure. Although the diameter stenosis of the side branch (evaluated visually by the investigators) was slightly lower than in previous studies, the $96 \%$ rate of angiographic follow-up reinforces the validity of the results, as does the fact that our study was multicentred with centralised angiographic analysis.

\section{Conclusions}

Use of a dedicated Nile PAX drug-eluting stent and of a new drugeluting balloon (DANUBIO) at the end of the procedure after kissing results in a low restenosis rate at six months in the side branch. Although suboptimal angiographic results obtained in the main 
branch were observed, they did not impact on the good clinical and angiographic results in the side branch. Additional and complementary studies with larger populations, combining newer-generation limus-eluting stents, which could enable better results on the main branch, along with the DANUBIO drug-eluting balloon would certainly be of especial interest. In this context, a sirolimuseluting version of the Nile dedicated stent platform is currently being developed.

\section{Impact on daily practice}

Restenosis following drug-eluting stent implantation in the treatment of bifurcation lesions remains a concern. The sixmonth angiographic results of the DEBSIDE trial show that drug-eluting balloon inflation using the DANUBIO balloon resulted in very low rates of restenosis of the side branch and, while further studies are warranted, its use may be a significant addition to the interventionalist's armamentarium by avoiding unnecessary side branch stenting in the treatment of all types of bifurcation lesion.

\section{Funding}

This study was supported by a grant from Minvasys.

\section{Conflict of interest statement}

J. Berland reports having received lecture honoraria and consulting fees from Minvasys. The other authors have no conflicts of interest to declare.

\section{References}

1. Ferenc M, Gick M, Kienzle RP, Bestehorn HP, Werner KD, Comberg T, Zhao M, Buettner HJ, Neumann FJ. Long-term outcome of percutaneous catheter intervention for de novo coronary bifurcation lesions with drug-eluting stents or bare-metal stents. Am Heart J. 2010;159:454-61.

2. Lassen JF, Holm NR, Stankovic G, Lefèvre T, Chieffo A, Hildick-Smith D, Pan M, Darremont O, Albiero R, Ferenc M, Louvard Y. Percutaneous coronary intervention for coronary bifurcation disease: consensus from the first 10 years of the European Bifurcation Club meetings. EuroIntervention. 2014;10:545-60.

3. Lee BK, Kim YH, Park DW, Yun SC, Ahn JM, Song HG, Lee JY, Kim WJ, Kang SJ, Lee SW, Lee CW, Lee JH, Seong IW, Park SW, Park SJ. Acute and long-term angiographic outcomes of side branch stenosis after randomized treatment of zotarolimus-, sirolimus-, and paclitaxel-eluting stent for coronary artery stenosis. J Korean Med Sci. 2012;27:1499-506.

4. Gao XF, Zhang YJ, Tian NL, Wu W, Li MH, Bourantas CV, Jiang XM, Wang ZM, Li B, Mao WX, Zhang JJ, Chen SL. Stenting strategy for coronary artery bifurcation with drug-eluting stents: a meta-analysis of nine randomised trials and systematic review. EuroIntervention. 2014;10:561-9.

5. Fanggiday JC, Stella PR, Guyomi SH, Doevendans PA. Safety and efficacy of drug-eluting balloons in percutaneous treatment of bifurcation lesions: the DEBIUT (drug-eluting balloon in bifurcaton Utrecht) registry. Catheter Cardiovasc Interv. 2008;71:629-35.

6. Belkacemi A, Agostoni P, Voskuil M, Stella PR. Coronary bifurcation lesions treated with the drug-eluting balloon: a preliminary insight from the DEBIUT study. EuroIntervention. 2011;7 Suppl K:K66-9.

7. Stella PR, Belkacemi A, Dubois C, Nathoe H, Dens J, Naber C, Adriaenssens T, van Belle E, Doevendans P, Agostoni P. A multicenter randomized comparison of drug-eluting balloon plus bare-metal stent versus bare-metal stent versus drug-eluting stent in bifurcation lesions treated with a single-stenting technique: sixmonth angiographic and 12 months clinical results of the drugeluting balloon in bifurcations trial. Catheter Cardiovasc Interv. 2012;80:1138-46.

8. Mathey DG, Wendig I, Boxberger M, Bonaventura K, Kleber FX. Treatment of bifurcation lesions with a drug-eluting balloon: the PEPCAD V (Paclitaxel Eluting PTCA Balloon in Coronary Artery Disease) trial. EuroIntervention. 2011;7 Suppl $\mathrm{K}: \mathrm{K} 61-5$.

9. López Minguez JR, Nogales Asensio JM, Doncel Vecino LJ, Sandoval J, Romany S, Martinez Romero P, Fernández Díaz JA, Fernández Portales J, González Fernández R, Martínez Cáceres G, Merchán Herrera A, Alfonso Manterola F; BABILON Investigators. A prospective randomised study of the paclitaxel-coated balloon catheter in bifurcated coronary lesions (BABILON trial): 24-month clinical and angiographic results. EuroIntervention. 2014;10:50-7.

10. Herrador JA, Fernandez JC, Guzman M, Aragon V. Drugeluting vs. conventional balloon for side branch dilation in coronary bifurcations treated by provisional $\mathrm{T}$ stenting. J Interv Cardiol. 2013;26:454-62.

11. Costa RA, Abizaid A, Abizaid AS, Garcia B, Berland J, Petrov I, Brenot P, van Guens RJ, Royer T, Rubino P, Lesiak M, Fajadet J; BIPAX Investigators. Procedural and early clinical outcomes of patients with de novo coronary bifurcation lesions treated with the novel Nile PAX dedicated bifurcation polymer-free paclitaxel coated stents: results from the prospective, multicentre, nonrandomised BIPAX clinical trial. EuroIntervention. 2012;7: 1301-9.

12. Afari EM, Granada JF. Mechanisms of Action in DrugCoated Balloons. Endovascular Today. 2012;August:53-58.

13. Kleber F, Mathey DG, Rittger H, Scheller B; German Drugeluting Balloon Consensus Group. How to use the drug-eluting balloon: recommendations by the German consensus group. EuroIntervention. 2011;7 Suppl K:K125-8.

14. Ramcharitar S, Onuma Y, Aben JP, Consten C, Weijers B, Morel MA, Serruys PW. A novel dedicated quantitative coronary analysis methodology for bifurcation lesions. EuroIntervention. 2008;3:553-7.

15. Girasis C, Schuurbiers JC, Onuma Y, Aben JP, Weijers B, Boersma E, Wentzel JJ, Serruys PW. Two-dimensional quantitative coronary angiographic models for bifurcation segmental analysis: in vitro validation of CAAS against precision manufactured plexiglas phantoms. Catheter Cardiovasc Interv. 2011;77:830-9. 
16. Steigen TK, Maeng M, Wiseth R, Erglis A, Kumsars I, Narbute I, Gunnes P, Mannsverk J, Meyerdierks O, Rotevatn S, Niemelä M, Kervinen K, Jensen JS, Galløe A, Nikus K, Vikman S, Ravkilde J, James S, Aarøe J, Ylitalo A, Helqvist S, Sjögren I, Thayssen P, Virtanen K, Puhakka M, Airaksinen J, Lassen JF, Thuesen L; Nordic PCI Study Group. Randomized study on simple versus complex stenting of coronary artery bifurcation lesions: the Nordic bifurcation study. Circulation. 2006;114:1955-61.

17. Hildick-Smith D, de Belder AJ, Cooter N, Curzen NP, Clayton TC, Oldroyd KG, Bennett L, Holmberg S, Cotton JM, Glennon PE, Thomas MR, Maccarthy PA, Baumbach A, Mulvihill NT, Henderson RA, Redwood SR, Starkey IR, Stables RH. Randomized trial of simple versus complex drug-eluting stenting for bifurcation lesions: the British Bifurcation Coronary study: old, new, and evolving strategies. Circulation. 2010;121:1235-43.

18. Chen SL, Santoso T, Zhang JJ, Ye F, Xu YW, Fu Q, Kan J, Paiboon C, Zhou Y, Ding SQ, Kwan TW. A randomized clinical study comparing double kissing crush with provisional stenting for treatment of coronary bifurcation lesions: results from the DKCRUSH-II (Double Kissing Crush versus Provisional Stenting Technique for Treatment of Coronary Bifurcation Lesions) trial. J Am Coll Cardiol. 2011;57:914-20.

19. Latib A, Colombo A, Castriota F, Micari A, Cremonesi A, De Felice F, Marchese A, Tespili M, Presbitero P, Sgueglia GA, Buffoli F, Tamburino C, Varbella F, Menozzi A. A randomized multicenter study comparing a paclitaxel drug-eluting balloon with a paclitaxel-eluting stent in small coronary vessels. The BELLO (Balloon Elution and Late Loss Optimization) Study. $J_{\text {Am Coll }}$ Cardiol. 2012;60:2473-80.

20. Scheller B, Hehrlein C, Bocksch W, Rutsch W, Haghi D, Böhm M, Speck U. Two year follow-up after treatment of coronary in-stent restenosis with a paclitaxel-coated balloon catheter. Clin Res Cardiol. 2008;97:773-81.

21. Hehrlein C, Richardt G, Wiemer M, Schneider H, Naber C, Hoffmann E, Dietz U. Description of Pantera Lux paclitaxel-releasing balloons and preliminary quantitative coronary angiography (QCA) results at six months in patients with coronary in-stent restenosis. EuroIntervention. 2011;7 Suppl K:K119-24.

22. Windecker S, Kolh P, Alfonso F, Collet JP, Cremer J, Falk V, Filippatos G, Hamm C, Head SJ, Jüni P, Kappetein AP,
Kastrati A, Knuuti J, Landmesser U, Laufer G, Neumann FJ, Richter DJ, Schauerte P, Sousa Uva M, Stefanini GG, Taggart DP, Torracca L, Valgimigli M, Wijns W, Witkowski A. 2014 ESC/ EACTS Guidelines on myocardial revascularization: The Task Force on Myocardial Revascularization of the European Society of Cardiology (ESC) and the European Association for CardioThoracic Surgery (EACTS) Developed with the special contribution of the European Association of Percutaneous Cardiovascular Interventions (EAPCI). EuroIntervention. 2015; 10:1024-94.

23. Pósa A, Nyolczas N, Hemetsberger R, Pavo N, Petnehazy O, Petrasi Z, Sangiorgi G, Gyöngyösi M. Optimization of drug-eluting balloon use for safety and efficacy: evaluation of the 2nd generation paclitaxel-eluting DIOR-balloon in porcine coronary arteries. Catheter Cardiovasc Interv. 2010;76:395-403.

24. Colombo A, Bramucci E, Saccà S, Violini R, Lettieri C, Zanini R, Sheiban I, Paloscia L, Grube E, Schofer J, Bolognese L, Orlandi M, Niccoli G, Latib A, Airoldi F. Randomized study of the crush technique versus provisional side-branch stenting in true coronary bifurcations: the CACTUS (Coronary Bifurcations: Application of the Crushing Technique Using Sirolimus-Eluting Stents) Study. Circulation. 2009;119:71-8.

25. Hanet C, Wijns W, Michel X, Schroeder E. Influence of balloon size and stenosis morphology on immediate and delayed elastic recoil after percutaneous transluminal coronary angioplasty. J Am Coll Cardiol. 1991;18:506-11.

26. Haude M, Erbel R, Issa H, Meyer J. Quantitative analysis of elastic recoil after balloon angioplasty and after intracoronary implantation of balloon-expandable Palmaz-Schatz stents. $J \mathrm{Am}$ Coll Cardiol. 1993;21:26-34.

27. Rensing BJ, Hermans WR, Strauss BH, Serruys P. Regional differences in elastic recoil after percutaneous transluminal coronary angioplasty: a quantitative angiographic study. $J$ Am Coll Cardiol. 1991;17:34B-38B.

28. Stone GW, Ellis SG, Cannon L, Mann JT, Greenberg JD, Spriggs D, O'Shaughnessy CD, DeMaio S, Hall P, Popma JJ, Koglin J, Russell ME; TAXUS V Investigators. Comparison of a polymer-based paclitaxel-eluting stent with a bare metal stent in patients with complex coronary artery disease: a randomized controlled trial. JAMA. 2005;294:1215-23. 